\title{
Gradhiva
}

GRADHIV

Revue d'anthropologie et d'histoire des arts

$33 \mid 2022$

Wampum : les perles de la diplomatie

\section{Lexique historique des termes}

\section{(2) OpenEdition}

1 Journals

Édition électronique

URL : https://journals.openedition.org/gradhiva/6170

DOI : 10.4000/gradhiva.6170

ISSN : 1760-849X

Traduction(s) :

Lexicon of Historic Terms - URL : https://journals.openedition.org/gradhiva/6280 [en]

Éditeur

Musée du quai Branly Jacques Chirac

Édition imprimée

Date de publication : 2 février 2022

Pagination : 158-163

ISBN : 978-2-35744-133-0

ISSN : 0764-8928

Référence électronique

"Lexique historique des termes », Gradhiva [En ligne], 33 | 2022, mis en ligne le 02 février 2022,

consulté le 14 septembre 2022. URL : http://journals.openedition.org/gradhiva/6170 ; DOI : https:// doi.org/10.4000/gradhiva.6170 
Lexique historique des termes

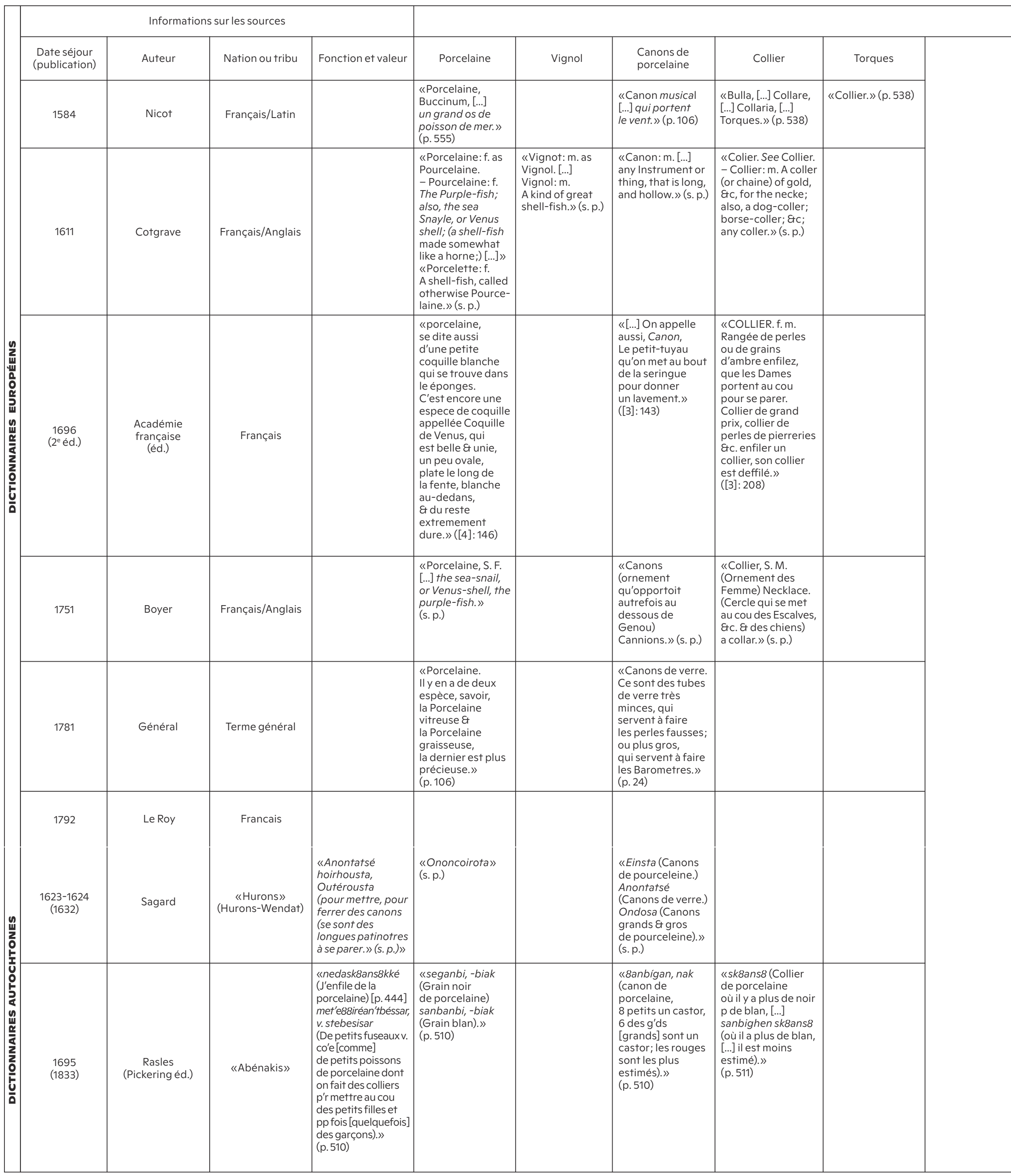




\section{Lexique historique des termes}

Explication des termes

\begin{tabular}{|c|c|c|c|c|c|c|c|}
\hline Baudrier & $\begin{array}{l}\text { Carcan/ } \\
\text { Quarquan }\end{array}$ & Ceinture & $\begin{array}{l}\text { Bastons } \\
\text { de porcelaine }\end{array}$ & $\begin{array}{c}\text { Brasse } \\
\text { de porcelaine }\end{array}$ & $\begin{array}{c}\text { Branche } \\
\text { de porcelaine }\end{array}$ & Patenostres & Rassade \\
\hline $\begin{array}{l}\text { «Balteus \& } \\
\text { Balteum.» (p. 79) }\end{array}$ & $\begin{array}{l}\text { «collier de fer } \\
\text { (au col).» (p. 596) }\end{array}$ & & & & & $\begin{array}{l}\text { «Recita precationem } \\
\text { dominicam.» (p. 523) }\end{array}$ & \\
\hline $\begin{array}{l}\text { «Baudrier: } \mathrm{m} .[\text {...]; } \\
\text { also, a belt, baudricke, } \\
\text { or sword-girdle of } \\
\text { that leather.» (s. p.) }\end{array}$ & $\begin{array}{l}\text { «Carquan: m. A } \\
\text { carcanet; a 5rich) } \\
\text { chaine for the } \\
\text { necke.» (s. p.) }\end{array}$ & $\begin{array}{l}\text { «Ceinture: Looke } \\
\text { Ceincture. } \\
\text { - Ceincture: f. } \\
\text { A girdle; also, the } \\
\text { waistband of a hose, } \\
\text { or doublet.» (s. p.) }\end{array}$ & $\begin{array}{l}\text { «Baston: m. A staffe, } \\
\text { bat, cudgel, } \\
\text { truncheon [...] prop, } \\
\text { supporter.» (s. p.) }\end{array}$ & $\begin{array}{l}\text { «Brasse: f. A fadome, } \\
\text { or arme-full; } \\
\text { or measure of fine } \\
\text { foot.» (s. p.) }\end{array}$ & $\begin{array}{l}\text { «Branche: f. [...] } \\
\text { Lineage; or, a } \\
\text { branch, or line, of a } \\
\text { Pettigree.» (s. p.) }\end{array}$ & $\begin{array}{l}\text { «Patenostre. la p. } \\
\text { The Pater-noster, } \\
\text { the Lords Prayer } \\
\text { Patenostrages: } \\
\text { m. Beads. } \\
\text { Patenostrier: m. } \\
\text { A maker, or seller } \\
\text { of beads [...] also, } \\
\text { beads; or a string, } \\
\text { list, or set of beads.» } \\
\text { (s. p.) }\end{array}$ & $\begin{array}{l}\text { «grains de verre } \\
\text { de couleur pers, } \\
\text { qu'ils appellant } \\
\text { rassades.» (Yves } \\
\text { d'Évreux sur le nord } \\
\text { du Brésil, 1615: 216) }\end{array}$ \\
\hline $\begin{array}{l}\text { «BAUDRIER. s. m. } \\
\text { Large ceinture qui } \\
\text { pend en escharpe, } \\
\text { \& sertà porter } \\
\text { l'espée. Bandrier } \\
\text { de cuir, bandrier en } \\
\text { broderie, donner des } \\
\text { coups de bandrier.» } \\
\text { ([3]: 92) }\end{array}$ & & $\begin{array}{l}\text { «CEINTURE. s. f. } \\
\text { Ce avec quoy se } \\
\text { ceint le corps. } \\
\text { Une ceinture d'or, } \\
\text { une ceinture } \\
\text { de diamants, } \\
\text { une ceinture avec } \\
\text { les pendans, ou avec } \\
\text { le porte-espée, } \\
\text { ceinture de cuir, } \\
\text { ceinture de haut de } \\
\text { chausse.» ([3]:153) }\end{array}$ & $\begin{array}{l}\text { «BASTON. s. m. Brin } \\
\text { de bois qu'on peut } \\
\text { tenir à la main.» } \\
\text { ([3]: 88) }\end{array}$ & $\begin{array}{l}\text { «BRASSE. s. f. } \\
\text { Mesure de la } \\
\text { longueur des deux } \\
\text { bras estendus, } \\
\text { Brasse de corde, } \\
\text { vingt brasses de } \\
\text { cords.» ([3]: 127) }\end{array}$ & $\begin{array}{l}\text { «BRANCHE. s. f. } \\
\text { Rameau d'un arbre, } \\
\text { le bois que pousse } \\
\text { le tronc d'un arbre. } \\
\text { Petite branche, } \\
\text { grosse branche.» } \\
\text { ([3]:126) }\end{array}$ & & \\
\hline \multirow[t]{4}{*}{$\begin{array}{l}\text { «Pendant, S. M. } \\
\text { les deux pendants } \\
\text { d'un Baudrier, } \\
\text { the bangers } \\
\text { of a Belt.» (s. p.) }\end{array}$} & & $\begin{array}{l}\text { «Ceinture, S. F. A } \\
\text { girdle, or sash, the } \\
\text { waste.» (s. p.) } 1751 \\
\text { (ceinturon = a waste- } \\
\text { belt) [aujourd'hui } \\
\text { waist belt] }\end{array}$ & $\begin{array}{l}\text { «Bâton, S. M, A stick, } \\
\text { staff, or [....].» (s. p.) }\end{array}$ & $\begin{array}{l}\text { «Brasse, S. F. A } \\
\text { fathom, a measure } \\
\text { of six foot.» (s. p.) }\end{array}$ & $\begin{array}{l}\text { «Branche (partie } \\
\text { de quelque chose) } \\
\text { a branch, or part of } \\
\text { any thing.» (s. p.) }\end{array}$ & & \\
\hline & & & & & & $\begin{array}{l}\text { «Patenoterie, } \\
\text { ou Patenottes. } \\
\text { On comprend sous } \\
\text { ce nom les Chapelets, } \\
\text { les Colliers de pierres } \\
\text { fausses, les Ouvrages } \\
\text { d'émail, les Perles } \\
\text { fausses, \&c.» (p. 96) }\end{array}$ & \\
\hline & & & & & & & $\begin{array}{l}\text { «Rassade, f. f. Perle } \\
\text { de verre don't on fait } \\
\text { des Chapelets.» } \\
\text { (p. 685) }\end{array}$ \\
\hline & & $\begin{array}{l}\text { «Ahouiche } \\
\text { (ceinture).» } \\
\text { (pas de wampum) } \\
\text { (s.p.) }\end{array}$ & & $\begin{array}{l}\text { «Escate téatan } \\
\text { (une brasse).» (s. p.) }\end{array}$ & & & \\
\hline $\begin{array}{l}\text { «nedans8ita'kámen } \\
\text { (baudrier, épee à la } \\
\text { ceinture).» (p. 439) }\end{array}$ & & $\begin{array}{l}\text { «pedeg8abis8n» } \\
\text { (pas de wampum) } \\
\text { [p. 405] } \\
\text { «k8natagat» (pas de } \\
\text { wampum) [p. 477] }\end{array}$ & & $\begin{array}{l}\text { «nekstaniganét } \\
\text { (une brasse).» } \\
\text { (p. 399) }\end{array}$ & $\begin{array}{l}\text { «sranakánnan } \\
\text { (branche).» (p. 399) }\end{array}$ & & \\
\hline
\end{tabular}


Lexique historique des termes

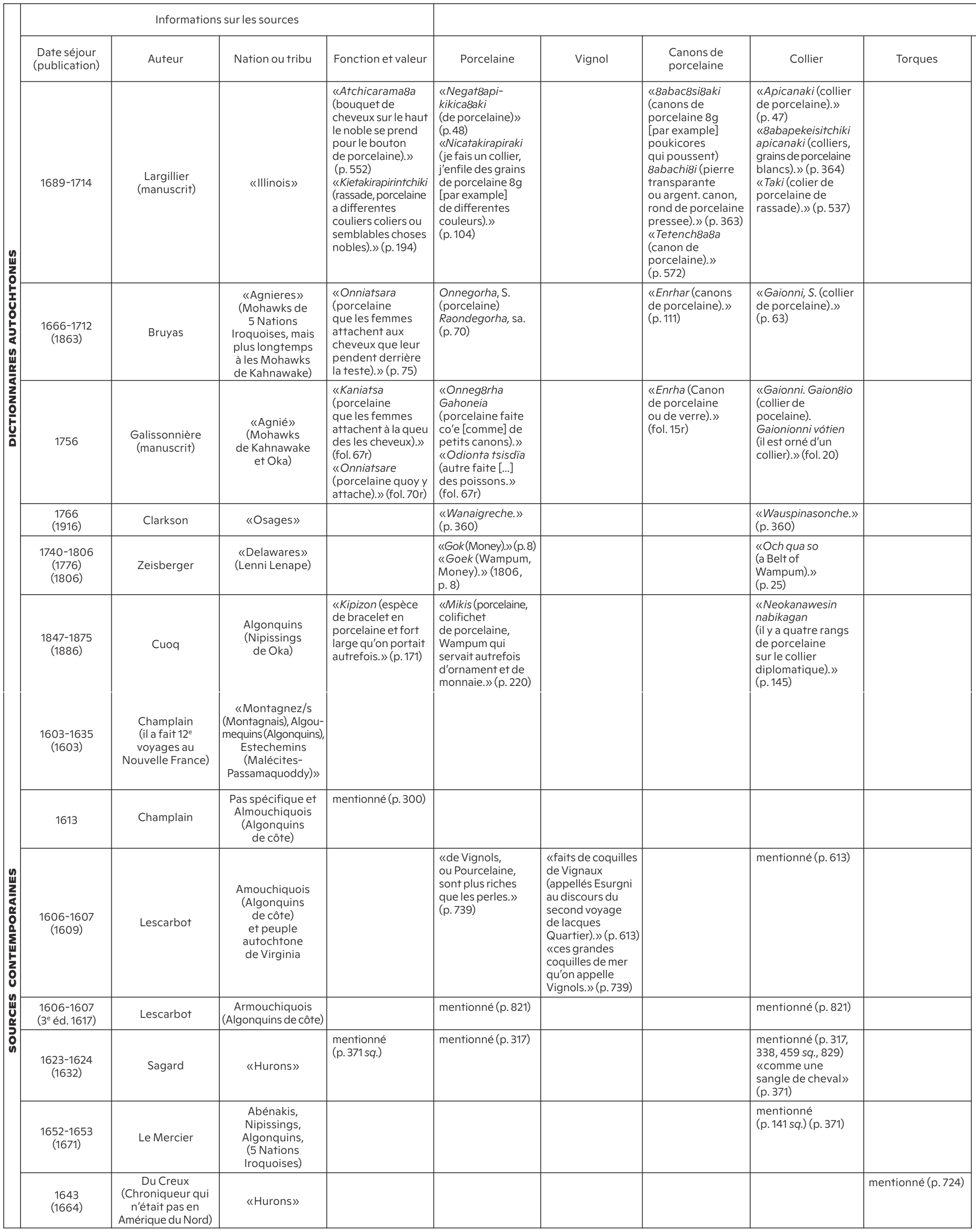




\section{Lexique historique des termes}

Explication des termes

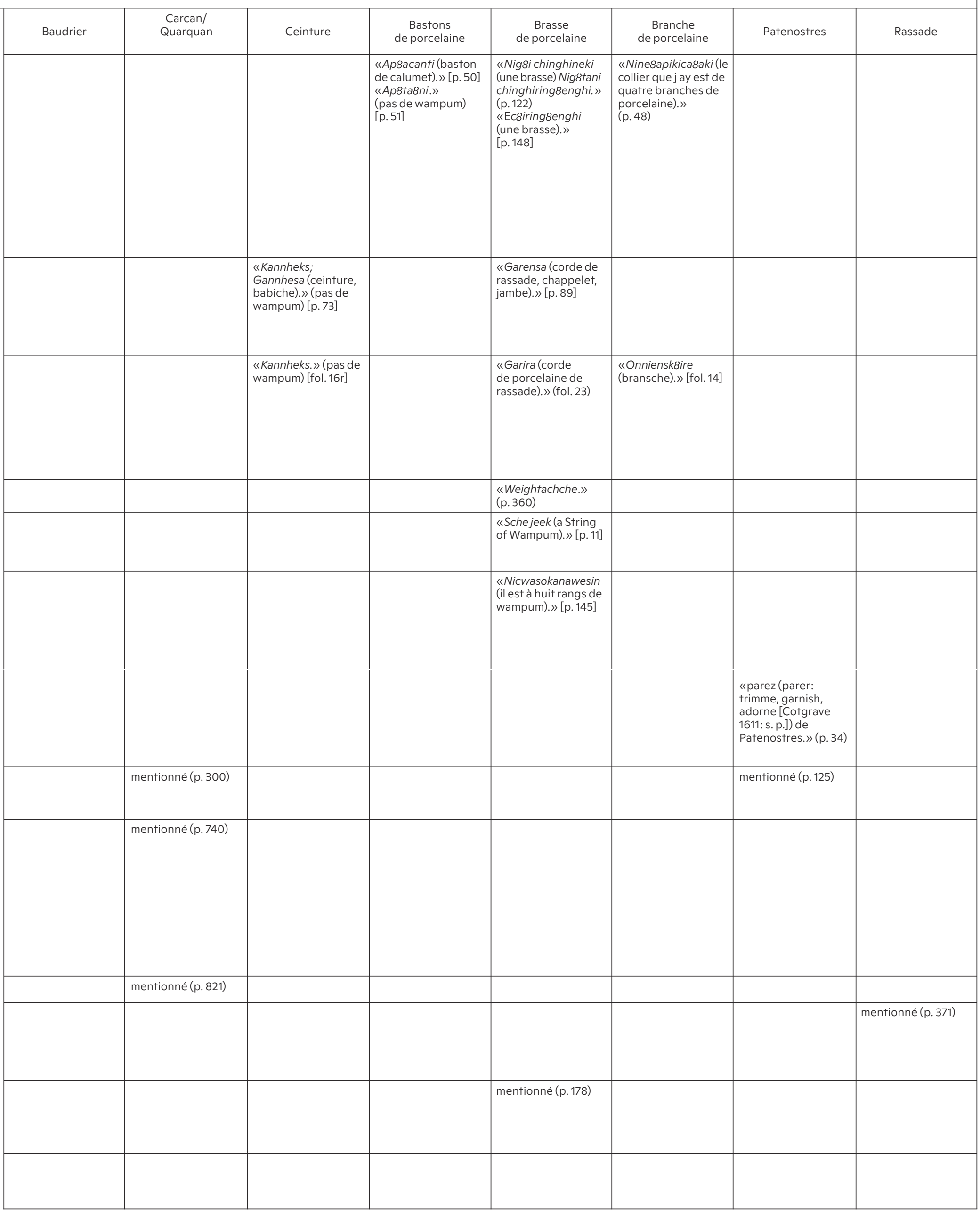


Lexique historique des termes

\begin{tabular}{|c|c|c|c|c|c|c|c|c|}
\hline \multicolumn{4}{|c|}{ Informations sur les sources } & \multirow[b]{2}{*}{ Porcelaine } & \multirow[b]{2}{*}{ Vignol } & \multirow[b]{2}{*}{$\begin{array}{l}\text { Canons de } \\
\text { porcelaine }\end{array}$} & \multirow[b]{2}{*}{ Collier } & \multirow[b]{2}{*}{ Torques } \\
\hline $\begin{array}{l}\text { Date séjour } \\
\text { (publication) }\end{array}$ & Auteur & Nation ou tribu & Fonction et valeur & & & & & \\
\hline 1673 (13 Juille & $\begin{array}{l}\text { Comte de } \\
\text { Frontenac }\end{array}$ & $\begin{array}{c}\text { « Ononontaguez } \\
\text { (Onondagas), } \\
\text { Agniez (Mohawks), } \\
\text { Onneioutes } \\
\text { (Oneidas), } \\
\text { Goïogouens } \\
\text { (Cayugas) et } \\
\text { Sonnontouans } \\
\text { (Senecas)» }\end{array}$ & & & & & mentionné (p. 213) & \\
\hline $\begin{array}{c}1679-1681 \\
(1876)\end{array}$ & $\begin{array}{l}\text { La Salle } \\
\text { (Margry éd.) }\end{array}$ & Miamis & & & & $\begin{array}{l}\text { «canons de } \\
\text { porcelaine et de } \\
\text { rassade.» (p.536) }\end{array}$ & & \\
\hline $\begin{array}{c}1679-1681 \\
(1876)\end{array}$ & $\begin{array}{l}\text { Sieur de Tonty } \\
\text { et sieur } \\
\text { de Boisrondet } \\
\text { (Margry éd.) }\end{array}$ & $\begin{array}{l}\text { «lllinois, } 5 \text { Nations } \\
\text { Iroquoises» }\end{array}$ & & & & & mentionné (p. 509) & \\
\hline $\begin{array}{c}\text { 1685, } 29 \text { janvie } \\
\text { (1959) }\end{array}$ & Bigot & Abénakis & & & & & & \\
\hline $\begin{array}{c}1698-1701 \\
(1722)\end{array}$ & $\begin{array}{l}\text { Baqueville } \\
\text { de la Potherie }\end{array}$ & $\begin{array}{l}\text { «5 Nations } \\
\text { Iroquoises, } \\
\text { Hurons, } \\
\text { Algonquins, } \\
\text { Nadouwessis } \\
\text { (Sioux del'est }) \text { ) }\end{array}$ & $\begin{array}{l}\text { mentionné ([1]: } \\
\text { 334) }\end{array}$ & $\begin{array}{l}\text { mentionné ([1]: } \\
\text { 333) }\end{array}$ & & & $\begin{array}{l}\text { mentionné ([1]: } \\
\text { 333) «Collier } \\
\text { de porcelaine.» } \\
\text { ([1]:334) } \\
\text { «Branches } \\
\text { de porcelaines.» } \\
\text { ([1]:334) }\end{array}$ & \\
\hline $\begin{array}{c}1699 \\
(1700)\end{array}$ & Massot (éd.) & Abénakis & & & & & mentionné (s. p.) & \\
\hline $\begin{array}{c}1711-1717 \\
(1724)\end{array}$ & Lafitau & $\begin{array}{l}\text { Agniés (Mohawks } \\
\text { de Kahnawake } \\
\text { données } \\
\text { mélangées avec } \\
\text { les informations } \\
\text { sur les Oneidas, } \\
\text { Onondagas, } \\
\text { Senecas et } \\
\text { Hurons-Wendat) }\end{array}$ & $\begin{array}{l}\text { mentionné ([1]: } \\
503 ;[2]: 55,58, \\
164,233,266)\end{array}$ & $\begin{array}{l}\text { mentionné ([1]: } \\
\text { 291, 491, 503 sqq.; } \\
{[2]: 332,341,414,} \\
432)\end{array}$ & & $\begin{array}{l}\text { «bien des canons } \\
\text { de porcelaine, qui } \\
\text { sont faits comme } \\
\text { des tuyaux de pipe } \\
\text { de Hollande.» } \\
\text { ([2]: 59) }\end{array}$ & $\begin{array}{l}\text { "collier ou bandes } \\
\text { de porcelaine } \\
\text { taillée en rond.» } \\
\text { ([1]: } 505 \text { sqq.; [2]: } \\
\text { 59) }\end{array}$ & \\
\hline $\begin{array}{l}1751 \\
(1908)\end{array}$ & $\begin{array}{l}\text { De Raymond } \\
\text { (Thwaites éd.) }\end{array}$ & $\begin{array}{c}\text { Saulteux } \\
\text { (Ojibwa de nord) }\end{array}$ & & & & & & \\
\hline $\begin{array}{c}1754 \\
(2007)\end{array}$ & $\begin{array}{l}\text { Bonin } \\
\text { (Galup éd.) }\end{array}$ & \begin{tabular}{|} 
«Hurons, Ottawas, \\
Sauteux, \\
Algonquins, \\
Potawatomies, \\
Outgamis or, \\
Miamis, \\
Mississaugas, \\
Mascoutens or Fire \\
Tribe, Puants \\
(Winnebago), Sioux, \\
Kickapoos, \\
Malomines or \\
Fallavoines \\
(Menominee), \\
Assiniboines, \\
Pawnees, and \\
Weas» (2007: 66)
\end{tabular} & & & & & $\begin{array}{l}\text { mentionné (fol. } 78 \\
\text { sq.) }\end{array}$ & \\
\hline $\begin{array}{c}1755 \\
21 \text { décembre } \\
(1756)\end{array}$ & Moreau & $\begin{array}{c}\text { Shawnee, Mingos, } \\
\text { Cherokees, Catawbas } \\
\text { (à fort Dusquene) }\end{array}$ & & & & & & \\
\hline $\begin{array}{c}1755-1760 \\
(1781)\end{array}$ & Pouchot & \begin{tabular}{|c|} 
Mississaugas \\
$(1760)$ \\
[Mohawks de \\
Kahnawake et Oka, \\
Abénakis et \\
Hurons-Wendat]
\end{tabular} & \begin{tabular}{|l|} 
«Ces femmes \\
sont chargées \\
de colliers, [...] \\
cesont des rubans \\
de porcelaine de \\
rasade.» ([3]: 236)
\end{tabular} & $\begin{array}{l}\text { «(marchandises } \\
\text { à Canada) de la } \\
\text { porcelaine [...] des } \\
\text { verroteries en } \\
\text { façon de porcelaine } \\
\text { d'un noir vineux.» } \\
\text { ([2]: } 300 \text { sq.) } \\
\text { mentionné ([3]: } \\
233 \text { sq., } 236 \text { sqq.) }\end{array}$ & & & $\begin{array}{l}\text { mentionné ([2]: } \\
219 \text { sq.) }\end{array}$ & \\
\hline 1762 & I'Abbée Arnaud & $\begin{array}{l}\text { Nouvelle-France, } \\
\text { pas spécifique }\end{array}$ & mentionné (p. 27) & mentionné (p. 26) & & & $\begin{array}{l}\text { mentionné(p. 27) } \\
\text { [information après } \\
\text { Bougainville] }\end{array}$ & \\
\hline 1763 & $\begin{array}{l}\text { Michel-Jean } \\
\text {-Hugues Péan }\end{array}$ & $\begin{array}{l}\text { Nouvelle-France, } \\
\text { pas spécifique }\end{array}$ & & & & & mentionné ( $p$. vii) & \\
\hline 1763 & Guillaume Estèbe & $\begin{array}{c}\text { Nouvelle-France, } \\
\text { pas spécifique }\end{array}$ & mentionné (p. 17) & mentionné (p. 16) & & & & \\
\hline $1771(1866)$ & Maurault & Abénakis & & & & & mentionné (p. 572) & \\
\hline
\end{tabular}


Lexique historique des termes

Explication des termes

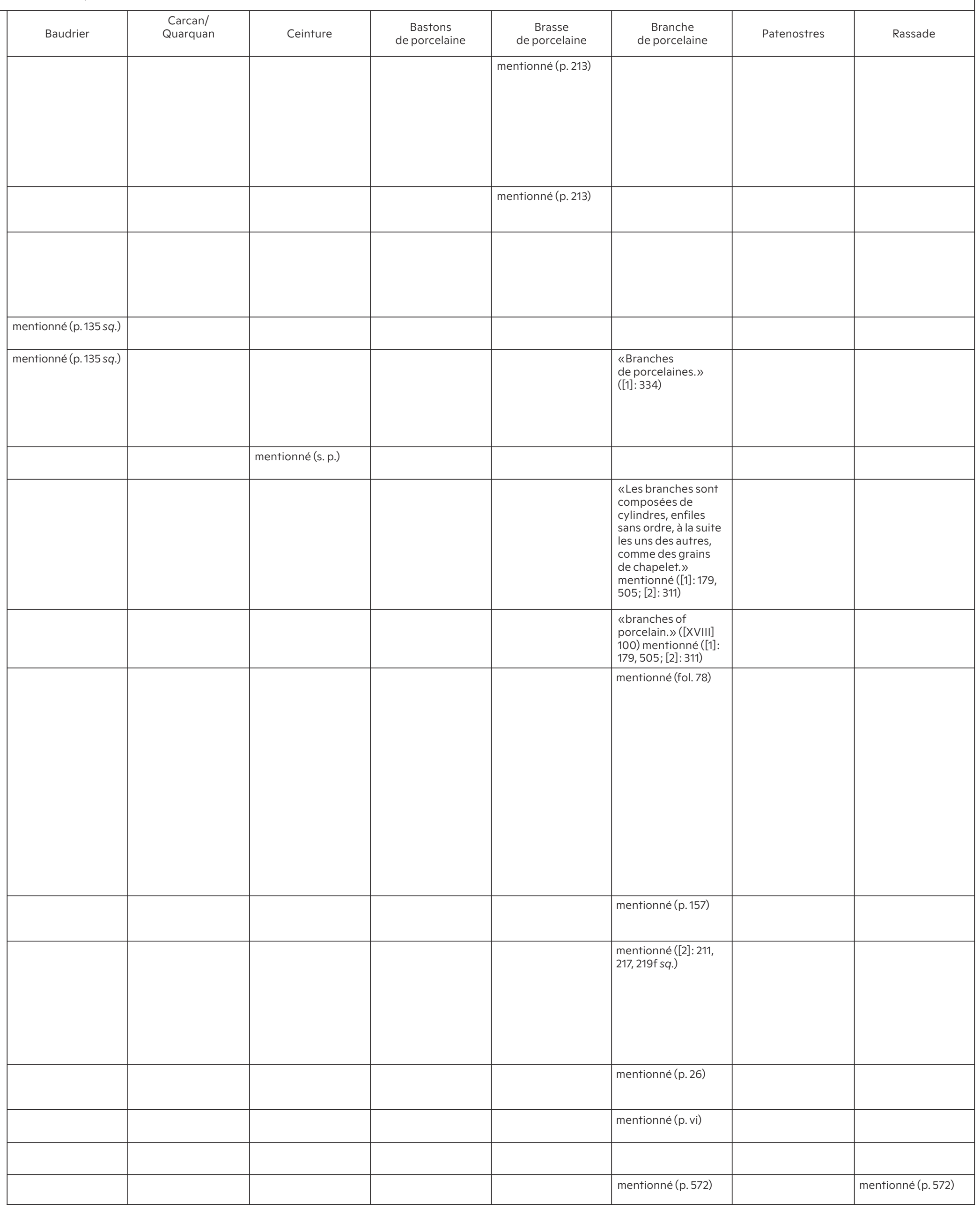

163 